\title{
The ability of the new ALBI scoring in predicting mortality, complications and prognostic comparison among cirrhotics
}

\author{
Iftikhar Haider Naqvi ${ }^{1}$, Abu Talib ${ }^{1}$, Khalid Mahmood ${ }^{1}$, Rida Abidi ${ }^{1}$, Saiyeda Nayema Zehra Rizvi ${ }^{2}$ \\ ${ }^{1}$ Department of Medicine, Dow University of Health Sciences, Karachi, Pakistan \\ ${ }^{2}$ Florence Medical Centre, Karachi, Pakistan
}

Gastroenterology Rev 2019; 14 (4): 250-257

DOI: https://doi.org/10.5114/pg.2019.83872

Key words: Child-Turcotte-Pugh score, Model for End stage Liver Disease, albumin-bilirubin.

Address for correspondence: Dr. Iftikhar Haider Naqvi, Department of Medicine, Dow University of Health Sciences, Karachi, Pakistan, phone: +92 3003655958, e-mail: drihnaqvi@gmail.com

\begin{abstract}
Introduction: Albumin-bilirubin (ALBI) is a newly devised scoring system for prognostication of liver cirrhosis. The ALBI has recently been validated and found superior to Child-Turcotte-Pugh score (CTP) and Model for End stage Liver Disease (MELD) in assessing severity of liver disease.

Aim: To determine the ALBI score's mortality prediction among cirrhotics, associated complications and to compare its prognostic proficiency to that of MELD and CTP.

Material and methods: The diagnostic accuracy of CTP, MELD and ALBI scores for mortality in hospitalized cirrhotic patients was determined by receiver operating characteristic curve (ROC) analysis. The areas under the ROC curve were calculated, with confidence intervals $(\mathrm{Cl})$ of $95 \%$. The best cut-off values were ascertained with the greatest specificity and sensitivity.

Results: The study showed overall in-hospital mortality of $25.5 \%$. Median CTP score was $10.2(\mathrm{IQR}=3)$ and area under curve (AUC) was 0.842 (95\% Cl: $0.817-0.868, p<0.001)$ with sensitivity of $75.0 \%$ and specificity of $79.2 \%$. Median MELD score was 20.9 (IQR $=7.2)$ and AUC was $0.836(95 \% \mathrm{Cl}: 0.810-0.863, p<0.001)$ with sensitivity of $76.6 \%$ and specificity of $76.7 \%$. Median ALBI score was $-1.1(\mathrm{IQR}=1.0)$, and AUC of ALBI was $0.852(95 \% \mathrm{Cl}: 0.826-0.879, p<0.001)$ with sensitivity and specificity of $78.1 \%$.

Conclusions: The objective prognostication and easy utilization of ALBI make it a useful alternative to MELD and CTP and therefore favour its applicability in clinical practice. Further validations in large prospective cohorts are needed for prognostic value of $A L B I$ in cirrhosis and its complications.
\end{abstract}

\section{Introduction}

Cirrhosis is an end-stage liver disease, characterized by eventual liver scarring by various chronic liver diseases progressing slowly over years to decades. Autopsy-based studied have reported global prevalence of $4.5 \%$ to $9.5 \%$ for cirrhosis among the general population [1-3]. Cirrhosis of the liver was ranked $14^{\text {th }}$ and $10^{\text {th }}$ as a cause of mortality worldwide and in developed countries, respectively, in 2001 [4]. It has been estimated that cirrhosis-related mortality will further escalate to rank $12^{\text {th }}$ as a cause of mortality worldwide by 2020 [5]. Pakistan occupies a huge burden of advanced stage of liver cirrhosis, where cirrhosis-related mortality/deaths (95\% uncertainty interval) increased from 10,324 $(6,129-16,651)$ to $31,373(16,325-61,028)$ from 1980 to 2010 [6]. Pakistan has a $21.7 \%$ to $27.5 \%$ age-standardized mortality rate (per 100,000) and a change in frequency for both genders from 1980 to 2010 [6]. A comprehensive treatment strategy for cirrhosis of the liver demands exact prognostication of disease, which would help to strengthen effective treatment modalities, even liver transplantation as a final rescue.

Various score systems, such as the Child-Turcotte score, Model for End stage Liver Disease (MELD), and Child-Turcotte-Pugh score (CTP), for assessment of prognosis among cirrhotic patients are being used in clinical settings. However, these scoring systems have several shortcomings that limit their prognostic implications. Although CTP scoring is easily applicable at bedside, its subjective nature for interpretation of ascites and encephalopathy and equal point allotment of all variables 
make its results highly variable. Another drawback of CTP scoring is the non-specific nature of albumin and bilirubin as severe sepsis and large volume ascites affect albumin level as well as bilirubin, which also becomes increased in sepsis, haemolysis and impaired renal function [7, 8]. Likely, ambiguous reference ranges for grading cirrhosis and its complications, such as ascites, bleeding, spontaneous bacterial peritonitis (SBP), and hepato-pulmonary syndrome, restricts the predictive accuracy of the MELD score. This prompts a need for evaluating other potential biomarkers to strengthen prognostication of cirrhosis and its complications.

Recently, the albumin-to-bilirubin (ALBI) score has emerged to evaluate severity of liver disease in hepatocellular carcinoma (HCC) [9]. The ALBI is a new and simple score, easily calculated at bedside without having to use subjective determinants such as ascites and encephalopathy. Its prognostic role in various liver diseases has recently been validated in several studies where it was found to be superior to CTP and MELD in assessing severity of liver disease especially in HBV-related cirrhosis [10-13].

\section{Aim}

This study aimed to determine the ability of ALBI score to predict mortality in cirrhosis, and its associated complication of SBP and compared its prognostic proficiency to that of MELD and CTP in our study population.

\section{Material and methods Patients}

This was a retrospective study where institutional review board approval was sought prior to commencement of the study. All confirmed cases of cirrhosis aged $\geq 18$ years of either sex, admitted to the department of medicine of Civil Hospital Karachi from April 2014 to August 2017 were enrolled in the study. For assessing and comparing the prognostic capability of ALBI to other scoring systems both compensated and decompensated liver cirrhosis (severity ranging from CTP class A to C) cases were included. Regardless of previous direct acting anti-viral or Interferon based treatment, all patients with viral $C$ hepatitis related cirrhosis were included in the study. Patients less than 18 years of age, those having comorbidities such as diabetes, renal disease, ischemic heart disease, etc., and those who left against medical advice were excluded from the study. Any case with incomplete information was also excluded. Demographic profiles including aetiology, clinical characteristics, liver chemistries and other biochemical details of patients were also retrieved. A total of 1254 patients were included in our study after a careful examination of each case per the above-mentioned crite- ria. The patients included had a minimum hospital stay of 3 days up to a maximum stay of 21 days.

\section{Cirrhosis}

Cirrhosis was identified based on clinical, radiological (ultrasonography or computed tomography) and biochemical parameters. Small shrunken liver and intra-abdominal varices with or without splenomegaly are important radiological parameters for cirrhosis. Liver cirrhosis was also proven on a histopathological basis, wherever required [14].

\section{Spontaneous bacterial peritonitis (SBP)}

Patients with cirrhosis were confirmed to have a diagnosis of spontaneous bacterial peritonitis when an ascitic fluid showed absolute polymorphonuclear leukocyte (PMN) count of at least 250 cells $/ \mathrm{mm}^{3}\left(0.25 \times 10^{9} / \mathrm{l}\right)$ and a positive ascitic fluid bacterial culture without an intra-abdominal surgically treatable source of infection [15]. Cirrhosis was assessed and staged for its severity in accordance with the Child-Pugh classification.

\section{Hepatocellular carcinoma}

The HCC was confirmed among cirrhotics with the help of advanced imaging either by three-phase computed tomography (CT) scan or magnetic resonance imaging (MRI). Alternatively HCC was also confirmed on CT scan/MRI by having contrast hyperenhancement in the arterial phase (wash-in) and hypoenhancement in the portal venous and/or delayed phases of acquisition (wash-out) among patients with liver cirrhosis with or without histopathological evidence) [16].

\section{Portal vein thrombosis (PVT)}

Non-malignant portal vein thrombosis was diagnosed by Doppler ultrasound with findings of hyperechoic material within the vessel lumen, lack of flow, and no evidence of dilatation of portal vein [17]. Further confirmation in terms of thrombus extent was made on contrast enhanced CT or MRI. Malignant portal vein thrombosis was confirmed through triphasic CT scan using criteria described by Shah et al. [18].

\section{Prognostic scoring system}

\section{Child-Turcotte-Pugh (CTP) scoring}

The score makes use of five clinical parameters (serum albumin, prothrombin time, ascites, hepatic encephalopathy and total bilirubin) related to chronic liver disease. Each parameter is scored 1-3, where a score of 3 designates the worst stage. The score ranges from 5 to 15 and is divided into three classes with CTP-A being a score from 5 to 6 , CTP-B ranging from 7 to 9 , and CTP -C having a range of 10 to 15 . 
Table I. Baseline characteristics of study participants

\begin{tabular}{|c|c|}
\hline Characteristics & Statistics $(N=1254)$ \\
\hline Age, mean (SD) [years] & $44.1(11.0)$ \\
\hline Gender, males, $n(\%)$ & $976(77.8)$ \\
\hline \multicolumn{2}{|l|}{ Etiology, $n(\%)$ : } \\
\hline $\mathrm{HCV}$ & $948(75.6)$ \\
\hline HBV & $188(15.0)$ \\
\hline Autoimmune & $38(3.0)$ \\
\hline Alcoholic & $32(2.6)$ \\
\hline Wilson & $20(1.6)$ \\
\hline PBC & $19(1.5)$ \\
\hline Cryptogenic & $5(0.4)$ \\
\hline Haemochromatosis & $4(0.3)$ \\
\hline \multicolumn{2}{|l|}{ Complications, $n$ (\%): } \\
\hline Hepatic encephalopathy & $1080(86.1)$ \\
\hline Ascites & $1068(85.2)$ \\
\hline Variceal bleeding & $556(44.3)$ \\
\hline Hyponatraemia $\left(\mathrm{Na}^{+}<130 \mathrm{mEq} / \mathrm{l}\right)$ & $489(39.0)$ \\
\hline SBP & $426(34.0)$ \\
\hline Portal vein thrombosis & $301(24.0)$ \\
\hline Hepatocellular carcinoma & $186(14.8)$ \\
\hline \multicolumn{2}{|l|}{ Baseline investigations, median (IQR): } \\
\hline Haemoglobin [g/dl] & $7.2(1.0)$ \\
\hline $\operatorname{TLC}\left[\times 10^{3} / \mu \mathrm{l}\right]$ & $22.8(9.1)$ \\
\hline INR & $1.8(0.6)$ \\
\hline Serum creatinine $[\mathrm{mg} / \mathrm{dl}]$ & $1.4(0.6)$ \\
\hline Serum bilirubin [mg/dl] & $4.0(3.1)$ \\
\hline Serum albumin $[\mathrm{g} / \mathrm{dl}]$ & $2.66(1.21)$ \\
\hline $\mathrm{ALT}[\mathrm{U} / \mathrm{l}]$ & $190.5(155.8)$ \\
\hline $\mathrm{ALP}[\mathrm{U} / \mathrm{I}]$ & $240.3(124.3)$ \\
\hline AFP $[\mathrm{IU} / \mathrm{ml}]$ & $213.0(180.0)$ \\
\hline \multicolumn{2}{|l|}{ Mortality scores, nedian (IQR): } \\
\hline CTP score & $10.2(3)$ \\
\hline MELD score & $20.9(7.2)$ \\
\hline ALBI score & $-1.1(1.0)$ \\
\hline
\end{tabular}

$H C V$ - hepatitis $C$ virus, $H B V$ - hepatitis $B$ virus, $P B C$ - primary biliary cholangitis, SBP - spontaneous bacterial peritonitis, TLC - total leukocyte count, INR - international normalized ratio, ALT - alanine aminotransferase, $A L P$-alkaline phosphatase, AFP - $\alpha$-fetoprotein, CTP-Child-Turcotte-Pugh, MELD - Model for End stage Liver Disease, ALBI - albumin-bilirubin.
Model for End stage Liver Disease (MELD) scoring

The MELD is another prognostic scoring system to determine severity of cirrhosis. MELD uses laboratory parameters (serum bilirubin, serum creatinine, and the international normalized ratio (INR)) for prediction of survival. The MELD score was calculated with the following equation: $\mathrm{MELD}=3.78 \times \ln$ [serum bilirubin $(\mathrm{mg} / \mathrm{dl})]+11.2 \times \ln [\mathrm{INR}]+9.57 \times \ln [$ serum creatinine $(\mathrm{mg} / \mathrm{dl})]+6.43$.

\section{Albumin-bilirubin (ALBI) score}

The ALBI score is a recently developed prognostic model for assessment of severity in liver disease. It is much simpler and objective. It was calculated using the following formula [9]: ALBI score $=-0.085 \times$ (serum albumin $g / l)+0.66 \times \lg ($ serum bilirubin $\mu \mathrm{mol} / \mathrm{l})$.

\section{Statistical analysis}

Means with standard deviations (SD) and medians with interquartile ranges (IQR) were determined for the continuous variables and analyzed. The categorical variables were reported as frequencies with percentages. As tests of significance, the Pearson $\chi^{2}$ test was used for categorical data, and independent sample $t$-test and Mann-Whitney $U$ test were used for continuous data as appropriate. The diagnostic accuracy of CTP, MELD and ALBI scores for mortality in hospitalized patients was determined by receiver operating characteristic curve (ROC) analysis. The areas under the ROC curve were calculated, with confidence intervals (CI) of $95 \%$. The best cut-off values were ascertained with the greatest specificity and sensitivity. Differences between the prognostic abilities of the three tests were compared using the Delong test. We used SPSS v.20 for data entry and analysis.

\section{Results}

\section{Baseline characteristics}

We included 1254 patients with chronic liver disease (CLD) who had a complete record in our system. As summarised in Table I, the mean age of our study population was $44.1 \pm 11$ years, with the majority of them being male ( $n=976,77.8 \%)$. More than three-quarters of the patients ( $n=948,75.6 \%$ ) had hepatitis C virus (HCV) infection followed by hepatitis B virus (HBV) $(n=$ $188,15 \%$ ), while the remainder had non-viral aetiology with autoimmune-related cirrhosis being the most common $(n=38,3.0 \%)$. The most frequent causes of admission were hepatic encephalopathy ( $n=1080,86.1 \%)$ and ascites ( $n=1068,85.1 \%)$, respectively.

The overall mortality in our population was of $25.5 \%$. We divided our population into surviving $(n=$ $934,74.5 \%)$ and non-surviving groups $(n=320,25.5 \%)$ and compared their clinical characteristics. The non-sur- 
viving patients were found to have a significantly higher TLC, serum creatinine, serum bilirubin and INR $(p<0.001)$. However, serum albumin was found to be much lower in the non- survivors $(p<0.001)$. The CTP, MELD and ALBI scores were also detected to be significantly higher in the non-survivors, as shown in Table II.

\section{Predictors of mortality in CLD}

This study evaluated the in-hospital mortality of CLD using ROC analyses for CTP, MELD and ALBI (Figure 1). The median CTP score was found to be 10.2 $(\mathrm{IQR}=3)$, which corresponds with Class $\mathrm{C}$ of the score. The AUC was calculated to be $0.842(95 \% \mathrm{Cl}$ : $0.817-$ $0.868, p<0.001$ ) with the best cut-off value at 11.05 having sensitivity of $75.0 \%$ and specificity of $79.2 \%$, respectively. The median MELD score came out at 20.9 $(\mathrm{IQR}=7.2)$ and the AUC was $0.836(95 \% \mathrm{Cl}: 0.810-$ $0.863, p<0.001)$. The best cut-off value was 22.9 with a sensitivity of $76.6 \%$ and specificity of $76.7 \%$.

The median ALBI score was $-1.1(\mathrm{IQR}=1.0)$, and it was found to be much higher in the non-survivors (median ALBI of -0.4 with IQR $=0.5$ ) than the survivors (median ALBI of $-1.3, I Q R=0.9$ ). The AUC of ALBI was the largest among the three at $0.852(95 \% \mathrm{Cl}:-0.826$ $-0.879, p<0.001$ ), having the best cut-off value at -0.832 with both sensitivity and specificity standing at $78.1 \%$. The difference between the three AUCs was not statistically significant (CTP-MELD, $p=0.855$; CTP-ALBI, $p=0.234$; MELD-ALBI, $p=0.420$ ).

We also performed the ROC analyses in the subgroup of patients having non-viral causes of CLD ( $n=118,9.4 \%$ ), comparing the prognostic abilities of the three scores (Figure 2). The AUC values of CTP, MELD and ALBI were 0.816 (95\% Cl: 0.737-0.896, $p<0.001)$, $0.704(95 \% \mathrm{Cl}: 0.604-0.805, p<0.001)$ and 0.873 (95\% Cl: $0.809-0.937, p<0.001$ ), respectively. The best cut-off values were found to be 10.9 (sensitivity $=79.2 \%$, specificity $=67.1 \%$ ) for CTP, 22.6 (sensitivity $=70.8 \%$, specificity $=65.7 \%$ ) for MELD and 0.958 (sensitivity $=83.3 \%$, specificity $=74.3 \%$ ) for ALBI. The AUC of ALBI came out to be significantly larger than that of MELD ( $p=0.013)$, while it was similar to CTP $(p=0.067)$. There was also no difference between AUCS of CTP and MELD ( $p=0.092)$.

\section{Analysis of patients presenting with complication of SBP}

We had 426 patients presenting with SBP. Among these, the majority had a viral cause of CLD ( $n=367$, $86.2 \%)$ and $14.3 \%(n=61)$ died during their hospital stay. The baseline characteristics of surviving and non-surviving groups are summarised in Table III. No difference was found in the ascitic fluid analysis, with almost the same count of PMN and similar ascitic albumin levels in both the groups. However, serum creatinine and bilirubin levels were higher in non-survivors $(p<0.001)$, while serum albumin was significantly lower in this group $(p<0.001)$. All the three prognostic scores were higher in the non-survivors and to compare their relative efficiencies in predicting in-hospital mortality in SBP, we performed ROC analysis. As shown in Figure 3, the AUCS for CTP, MELD and ALBI were $0.887(95 \% \mathrm{Cl}$ : $0.836-0.939, p<0.001), 0.793(95 \% \mathrm{Cl}: 0.726-0.861$, $p<0.001)$ and 0.908 (95\% Cl: 0.864-0.952, $p<0.001)$,

Table II. Comparison of clinical characteristics between surviving and non-surviving patients with liver cirrhosis $(N=1254)$

\begin{tabular}{|c|c|c|c|}
\hline Parameter & Surviving patients $(n=934)$ & Non-surviving patients $(n=320)$ & $P$-value \\
\hline Age, mean (SD) [years] & $43.8(11.1)$ & $44.9(10.8)$ & 0.138 \\
\hline Gender, males, $n(\%)$ & $731(78.3)$ & $245(76.6)$ & 0.527 \\
\hline Viral etiology, $n(\%)$ & $864(92.5)$ & $272(85.0)$ & $<0.001$ \\
\hline TLC, median $(\mathrm{IQR})\left[\times 10^{3} / \mu \mathrm{I}\right]$ & $22.5(8.6)$ & $23.2(12.8)$ & $<0.001$ \\
\hline INR, median (IQR) & $1.7(0.7)$ & $2.0(0.6)$ & $<0.001$ \\
\hline Serum creatinine, median (IQR) [mg/dl] & $1.3(0.5)$ & $1.9(1.5)$ & $<0.001$ \\
\hline Serum bilirubin, median (IQR) [mg/dl] & $3.5(3.1)$ & $5.2(2.6)$ & $<0.001$ \\
\hline Serum albumin, median (IQR) $[\mathrm{g} / \mathrm{dl}]$ & $2.9(1.0)$ & $1.9(0.7)$ & $<0.001$ \\
\hline ALT, median (IQR) [U/I] & $188.1(147.1)$ & $197.6(191.6)$ & 0.113 \\
\hline ALP, median (IQR) [U/I] & $244.0(122.9)$ & $231.8(127.8)$ & 0.089 \\
\hline CTP score, median (IQR) & $9.75(3)$ & $12.0(2)$ & $<0.001$ \\
\hline MELD score, median (IQR) & $19.7(5.8)$ & $26.8(6.8)$ & $<0.001$ \\
\hline ALBI score, median (IQR) & $-1.3(0.9)$ & $-0.4(0.5)$ & $<0.001$ \\
\hline
\end{tabular}

$P$-value of $<0.05$ is considered significant. TLC - total leukocyte count, INR - international normalized ratio, ALT - alanine aminotransferase, CTP - ChildTurcotte-Pugh, MELD - Model for End stage Liver Disease, ALBI - albumin-bilirubin. 
A

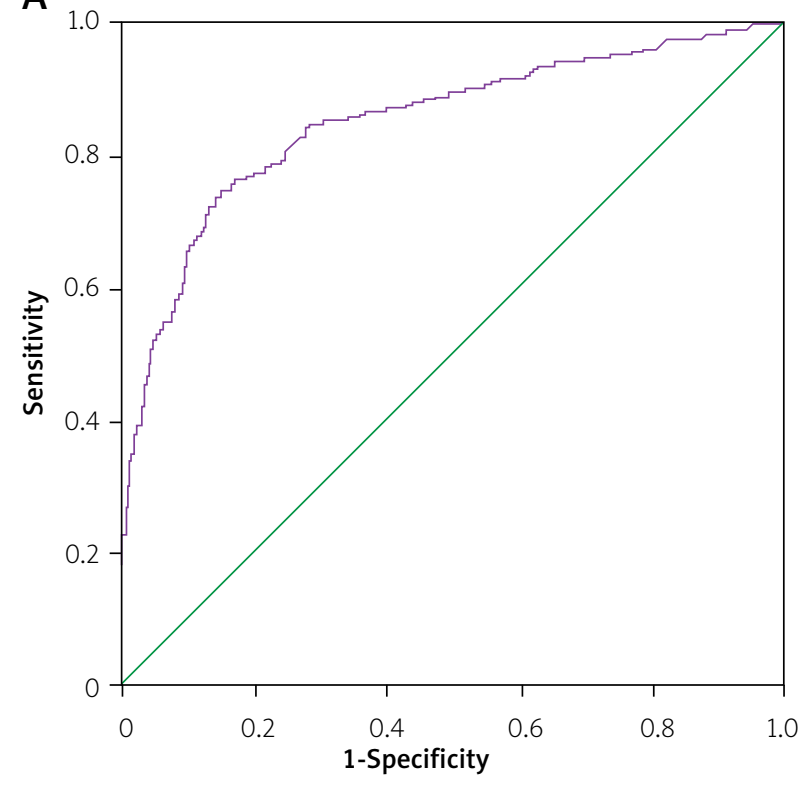

B

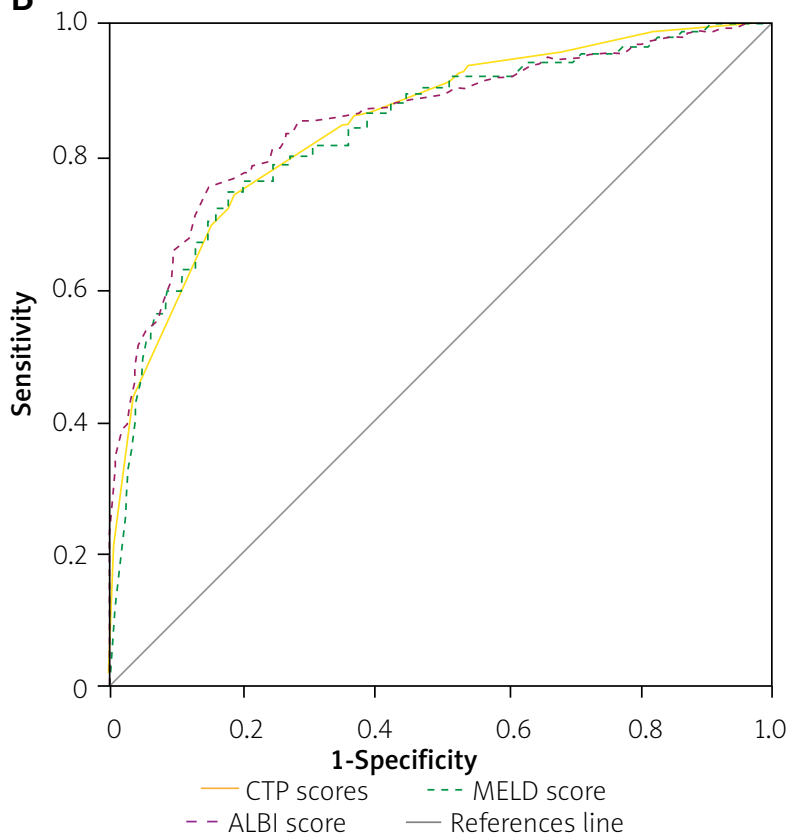

Figure 1. A - Receiver operating curve (ROC) analysis of ALBI score for predicting in-hospital mortality of cirrhotic patients. B - A comparison of the prognostic abilities of Child-Turcotte-Pugh (CTP), Model for End stage Liver Disease (MELD) and albumin-bilirubin (ALBI) scores is also presented

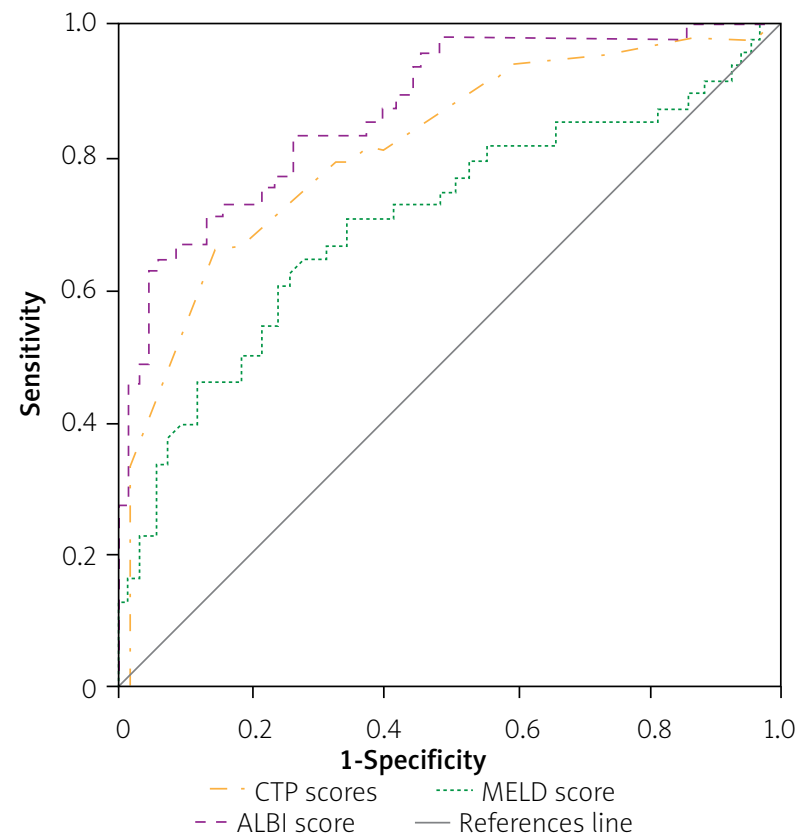

Figure 2. Receiver operating curve (ROC) indicating the relative efficiencies for predicting in hospital mortality of cirrhotic patients with non-viral aetiology by Child-Turcotte-Pugh (CTP), Model for End stage Liver Disease (MELD) and albumin-bilirubin (ALBI) scores respectively. The best cut-off value for CTP was 11.5 (sensitivity $=78.7 \%$, specificity $=87.4 \%$ ). MELD had the best cut-off value at 22.0 (sensitivity $=70.5 \%$, specificity $=79.7 \%$ ), while for ALBI it was -0.616 (sensitivity $=83.6 \%$, specificity $=84.9 \%$ ). The AUCs of CTP and $A L B I$ were found to be significantly larger than those of MELD (CTP-MELD, $p=0.013$; MELD-ALBI, $p=0.006$ ), but there was no statistical significance for the comparison of CTP versus ALBI $(p=0.196)$.

\section{Analysis of patients presenting with complication of HCC}

A total of 186 patients presented with HCC. More than half of these had HCV infection $(n=105,56.5 \%)$ and approximately two-thirds $(n=125,67.2)$ died during the hospital stay. The ROC analysis done to predict in-hospital mortality revealed similar results, with no significant differences among the three scores. As depicted in Figure 4, the AUC for CTP was 0.750 (95\% Cl: $0.678-0.823, p<0.001$ ) with the best cut-off value at 11.4 (sensitivity $=78.4 \%$, specificity $=62.3 \%$ ). For MELD, the AUC was 0.770 (95\% Cl: 0.701-0.839, $p<0.001$ ) and the best cut-off value was 22.1 (sensitivity $=74.4 \%$, specificity $=65.6 \%$ ). The ALBI score had an AUC of $0.780(95 \% \mathrm{Cl}:-0.710-0.849, p<0.001)$ with the best cut-off value at -0.565 (sensitivity $=72.8 \%$, specificity $=67.2 \%$ ). 
Table III. Clinical characteristics of surviving and non-surviving patients presenting with complication of spontaneous bacterial peritonitis $(N=426)$

\begin{tabular}{|c|c|c|c|}
\hline Parameter & Surviving patients $(n=365)$ & Non-surviving patients $(n=61)$ & $P$-value \\
\hline Age, mean (SD) [years] & $43.3(10.8)$ & $44.3(9.5)$ & 0.500 \\
\hline Gender, males, $n$ (\%) & $317(86.8)$ & $50(82.0)$ & 0.307 \\
\hline Viral etiology, $n(\%)$ & $339(92.9)$ & $50(82.0)$ & 0.005 \\
\hline Ascitic fluid PMN, median (IQR) [cell/ml] & $299.5(47.3)$ & $290.0(46.5)$ & 0.158 \\
\hline Ascitic fluid albumin, median (IQR) [g/dl] & $1.5(0.3)$ & $1.5(0.3)$ & 0.920 \\
\hline INR, median (IQR) & $1.7(0.6)$ & $1.7(0.5)$ & 0.855 \\
\hline Serum creatinine, median $(\mathrm{IQR})[\mathrm{mg} / \mathrm{dl}]$ & $1.2(0.4)$ & $1.9(1.5)$ & $<0.001$ \\
\hline Serum bilirubin, median (IQR) $[\mathrm{mg} / \mathrm{dl}]$ & $3.6(2.7)$ & $5.2(2.3)$ & $<0.001$ \\
\hline Serum albumin, median (IQR) $[\mathrm{g} / \mathrm{dl}]$ & $2.8(1.0)$ & $1.8(0.4)$ & $<0.001$ \\
\hline ALT, median (IQR) [U/I] & $185.4(142.3)$ & $148.1(176.8)$ & 0.110 \\
\hline CTP score, median (IQR) & $9.0(4)$ & $13.0(1)$ & $<0.001$ \\
\hline MELD score, median (IQR) & $18.7(5.7)$ & $24.4(7.6)$ & $<0.001$ \\
\hline ALBI score, median (IQR) & $-1.3(0.9)$ & $-0.3(0.3)$ & $<0.001$ \\
\hline
\end{tabular}

$P$-value of $<0.05$ is considered significant. PMN - polymorphonuclear leukocytes, INR - international normalized ratio, ALT - alanine aminotransferase, CTP-Child-Turcotte-Pugh, MELD - Model for End stage Liver Disease, ALBI-albumin-bilirubin.

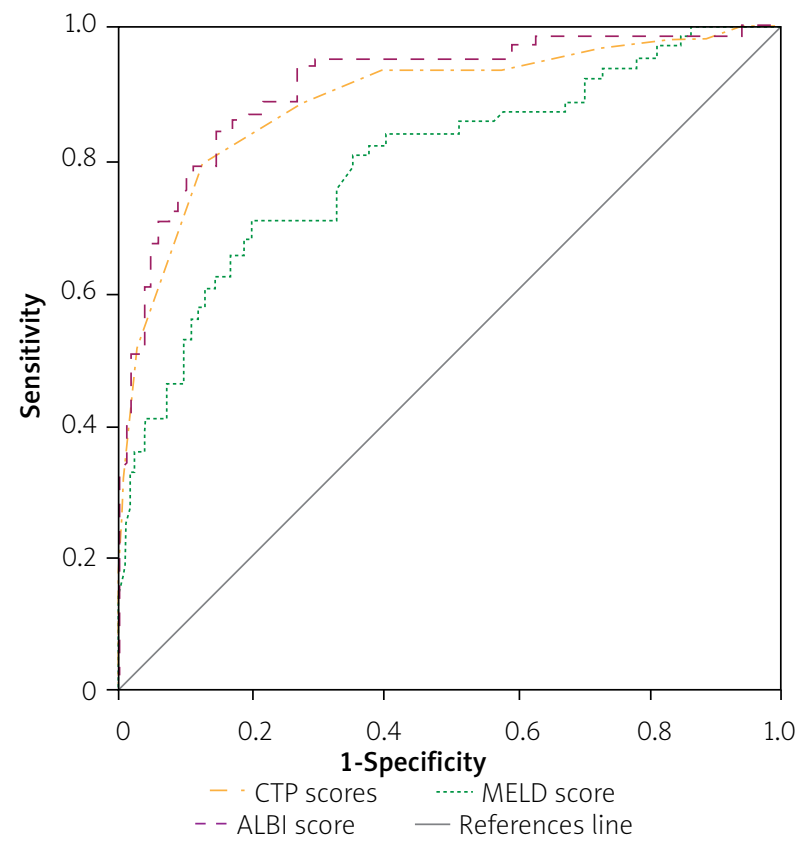

Figure 3. Receiver operating curve (ROC) indicating the relative efficiencies for predicting in-hospital mortality of cirrhotic patients, presenting with complication of spontaneous bacterial peritonitis (SBP), by Child-Turcotte-Pugh (CTP), Model for End stage Liver Disease (MELD) and albumin-bilirubin (ALBI) scores

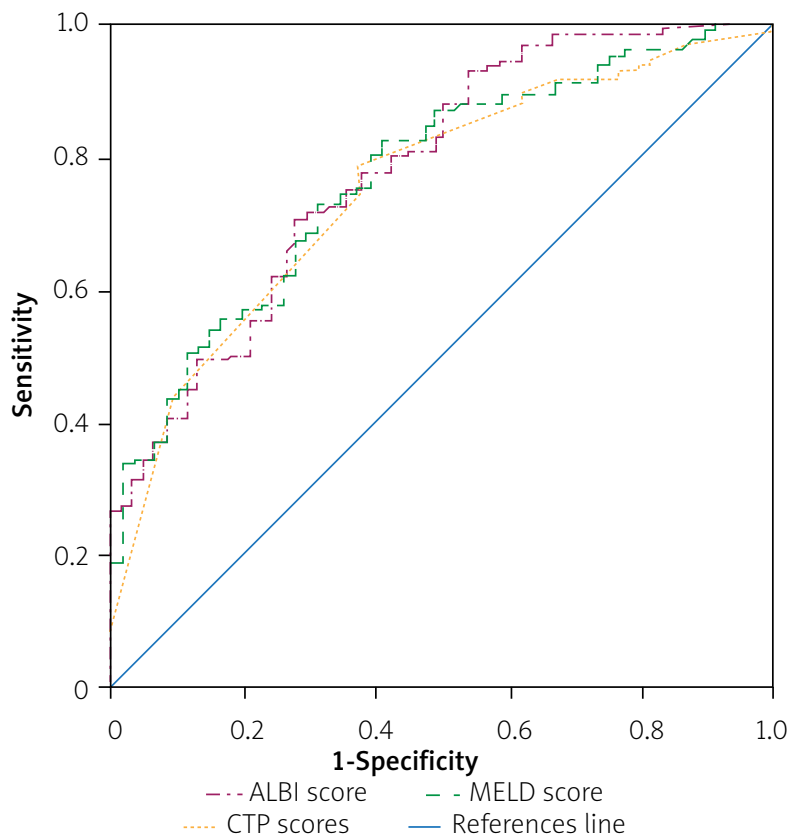

Figure 4. Receiver operating curve (ROC) indicating the relative efficiencies for predicting in-hospital mortality of cirrhotic patients, presenting with hepatocellular carcinoma, by Child-Turcotte-Pugh (CTP), Model for End stage Liver Disease (MELD) and albumin-bilirubin (ALBI) scores 


\section{Discussion}

The majority of patients (77.8\%) in this study were male; this male predominance is similar to an earlier study [14]. Mean age of $44.1 \pm 11$ years among our patient population is in accordance with earlier published studies. Viral-related aetiology, hepatitis C (75.6\%) and hepatitis B (15\%) dominate in this study, which is comparable to previously published studies [14, 19]. The overall mortality of $25.5 \%$ in our study group was congruent with the mortality rate of CLD patients of a previous study conducted by Naqvi et al. in the same hospital [20].

Currently CTP and MELD are the most commonly used prognostic models for assessing liver dysfunction, and several studies have been conducted to compare their prognostic efficiencies [21]. Recently the CTP score was found to be suitable for prediction of possible blood loss during transplantation of the liver [22]. With highly subjective assessment of ascites and encephalopathy by the scorer, the predictive accuracy of CTP may be variable [23]. Although MELD has been used as a standard tool for liver transplantation donor allocation and assessing 3- to 6-month survival in liver failure patients $[24,25]$, it has failed to accurately predict survival in $15 \%$ to $20 \%$ of patients [26]. Furthermore, both these scoring systems have limited diagnostic accuracy in conditions associated with liver cirrhosis such as hepato-renal syndrome, ascites, SBP, bleeding etc. In a recent study by Hassan et al., CTP and MELD were found to have shortcomings in accurately predicting in-hospital mortality of SBP patients [27].

On the other hand, ALBI has been a novel development in analysing mortality of liver failure in hepatocellular carcinoma [9]. It is a simple score, making use of serum albumin and total bilirubin as prognostic markers in liver dysfunction. Many studies have shown ALBI to have higher predictive power than CTP and MELD [28, 29]. In this study, however, ALBI had just as good prognostic efficacy of mortality associated with hepatocellular carcinoma in cirrhosis $(p<0.001)$ as MELD and CTP scores. This contrasts with earlier studies where ALBI was found to be superior to CTP and MELD $[9,30]$. Studies have also been undertaken to evaluate prognostic efficiency of ALBI in other liver diseases [10-13]. Shao et al. found ALBI to be an equally significant score of mortality in cirrhosis as MELD and CTP [31]. Chen et al. concluded ALBI to be superior to CTP and MELD in assessing HBV-associated cirrhosis [10].

This study found that non-surviving patients had a much higher ALBI score than the survivors and showed ALBI to be a good predictor of in-hospital mortality of liver cirrhosis $(p<0.001)$ with similar efficiency as MELD and CTP. Predictive ability of ALBI in a non-viral aetiology of cirrhosis such as autoimmune hepatitis, however, was significantly higher than MELD $(p=0.013)$. This indicates serum albumin and total bilirubin to have important prognostic implications and $A L B I$ to be an effective alternative in assessing severity of chronic liver diseases of various non-viral aetiologies as well. Among subsets of the patient population, spontaneous bacterial peritonitis (SBP) and HCC were also analysed in this study. Both CTP $(p=0.013)$ and ALBI $(p=0.006)$ were found to be superior predictors of mortality in SBP compared to MELD. Non-surviving patients with SBP had a similar ascitic fluid PMN count and ascitic fluid albumin, but a much higher serum creatinine and total bilirubin and significantly lower serum albumin $(p<0.001)$. Therefore, these biomarkers seem to be important players in progression of complications of liver cirrhosis and should be further studied in relation to prognosis of SBP and other complications.

Albumin is synthesized by the liver, and low serum albumin is especially pivotal in mortality associated with liver failure. Albumin has known benefits in management of cirrhosis and diseases related to it [32]. In this study, albumin was found to be consistently low in non-surviving patients across all subgroups. This might explain a stronger prognostic ability of CTP and ALBI than MELD in our subgroups of patients with SBP and non-viral aetiology, as these two make use of serum albumin in their diagnostic method. Perhaps addition of albumin to the diagnostic criteria of MELD may further strengthen the prognostication power of the score.

Our study had several limitations. First, it was a retrospective study causing a bias in patient selection and limited availability of data. Secondly, the sample size of the subgroup of non-viral aetiology was small and further studies with a larger sample should be conducted to validate the findings. Third, we based our study on a single value of ALBI. A more dynamic assessment of ALBI should be made throughout the hospital course of patients to better understand the role of ALBI in assessing severity of liver cirrhosis.

\section{Conclusions}

The ALBI is a simple prognostic test that can be calculated at bedside using only two biomarkers that are readily available in routine blood tests. This can help clinicians in faster medical decision making and greatly improve patient outcome. Its strong objective prognostication and easy utilization make it a useful alternative to MELD and CTP, and therefore its application should be widely fostered in our clinical practice. More prospective studies with larger cohorts across multiple tertiary hospitals should be conducted to validate the prognostic value of ALBI in cirrhosis and its wide array of complications. 


\section{Conflict of interest}

The authors declare no conflict of interest.

\section{References}

1. Melato M, Sasso F, Zanconati F. Liver cirrhosis and liver cancer. A study of their relationship in 2563 autopsies. Zentralbl Pathol 1993; 139: 25-30.

2. Graudal N, Leth P, Marbjerg L, et al. Characteristics of cirrhosis undiagnosed during life: a comparative analysis of 73 undiagnosed cases and 149 diagnosed cases of cirrhosis, detected in 4929 consecutive autopsies. J Intern Med 1991; 230: 165-71.

3. Lim YS, Kim WR. The global impact of hepatic fibrosis and endstage liver disease. Clin Liver Dis 2008; 12: 733-46.

4. Mathers C, Lopez A, Murray C. The burden of disease and mortality by condition: data, methods, and results for 2001. In: Global burden of disease and risk factors. Lopez A, Mathers C, Ezzati M, et al. (eds.). Oxford University Press and the World Bank, Washington (DC) 2006; 45-93.

5. Murray CJ, Lopez AD. Alternative projections of mortality and disability by cause 1990-2020: global burden of disease study. Lancet 1997; 349: 1498-504.

6. Mokdad AA, Lopez AD, Shahraz S, et al. Liver cirrhosis mortality in 187 countries between 1980 and 2010: a systematic analysis. BMC Med 2014; 12: 145.

7. Fleck A, Raines G, Hawker F, et al. Increased vascular permeability: a major cause of hypoalbuminaemia in disease and injury. Lancet 1985; 1: 781-4.

8. Henriksen JH, Parving HH, Christiansen L, et al. Increased transvascular escape rate of albumin during experimental portal and hepatic venous hypertension in the pig. Relation to findings in patients with cirrhosis of the liver. Scand J Clin Lab Invest 1981; 41: 289-99.

9. Johnson PJ, Berhane S, KagebayashiC, et al. Assessment of liver function in patients with hepatocellular carcinoma: a new evidence-based approach-the ALBI grade. J Clin Oncol 2015; 33: 550-8.

10. Chen RC, Cai YJ, Wu JM, et al. Usefulness of albumin-bilirubin grade for evaluation of long-term prognosis for hepatitis B-related cirrhosis. J Viral Hepat 2017; 24: 238-45.

11. Chan AW, Chan RC, Wong GL, et al. New simple prognostic score for primary biliary cirrhosis: albumin-bilirubin score. J Gastroenterol Hepatol 2015; 30: 1391-6.

12. Zou D, Qi X, Zhu C, et al. Albumin-bilirubin score for predicting the in-hospital mortality of acute upper gastrointestinal bleeding in liver cirrhosis: a retrospective study. Turk J Gastroenterol 2016; 27: 180-6.

13. Peng Y, Qi X, Tang S, et al. Child-Pugh, MELD, and ALBI scores for predicting the in-hospital mortality in cirrhotic patients with acute-on-chronic liver failure. Expert Rev Gastroenterol Hepatol 2016; 10: 971-80.

14. Naqvi I, Mahmood K, Talib A, et al. Infections in cirrhotics: types, microbiological spectrum and risk factors - 5-year cohort study. Open J Gastroenterol 2014; 4: 105-17.

15. Dever JB, Sheikh MY. Review article: spontaneous bacterial peritonitis: bacteriology, diagnosis, treatment, risk factors and prevention. Aliment Pharmacol Ther 2015; 41: 1116-31.

16. Hussain K, El-Serag HB. Epidemiology, screening, diagnosis and treatment of hepatocellular carcinoma. Minerva Gastroenterol Dietol 2009; 55: 123-38.
17. Tarantino L, Ambrosino P, Di Minno MN. Contrast-enhanced ultrasound in differentiating malignant from benign portal vein thrombosis in hepatocellular carcinoma. World J Gastroenterol 2015; 21: 9457-60.

18. Shah ZK, McKernan MG, Hahn PF, Sahani DV. Enhancing and expansile portal vein thrombosis: value in the diagnosis of hepatocellular carcinoma in patients with multiple hepatic lesions. AJR Am J Roentgenol 2007; 188: 1320-3.

19. Naqvi IH, Mahmood K, Salekeen S, et al. Determining the frequency and severity of malnutrition and correlating it with the severity of liver cirrhosis. Turk J Gastroenterol 2013; 24: 415-22.

20. Naqvi IH, Mahmood K, Talib A, et al. Spectrum of diseases, disease related mortality and overall financial burden of hospitalized patients in medical wards of tertiary care public hospital. Open J Epidemiol 2017; 7: 337-48.

21. Peng Y, Qi X, Guo X. Child-Pugh versus MELD Score for the assessment of prognosis in liver cirrhosis: a systematic review and meta-analysis of observational studies. Medicine (Baltimore) 2016; 95: e2877.

22. Rybicka A, Bessas M, Gałek J, et al. Predicting intraoperative blood loss based on the scale of liver failure assessment. Piel Chir Angiol 2018; 12: 147-52.

23. Durand F, Valla D. Assessment of prognosis of cirrhosis. Semin Liver Dis 2008; 28: 110-22.

24. Gambato M, Senzolo M, Canova D, et al. Algorithm for prioritization of patients on the waiting list for liver transplantation. Transplant Proc 2007; 39: 1855-6.

25. Freeman RB Jr, Wiesner RH, Harper A, et al. The new liver allocation system: moving toward evidence-based transplantation policy. Liver Transpl 2002; 8: 851-8.

26. Kamath P, Kim W. The model for end-stage liver disease (MELD). Hepatology 2007; 45: 797-805.

27. Hassan E, Abdel Rehim AS. Creatinine modified Child-TurcottePugh and integrated model of end-stage liver disease scores as predictors of spontaneous bacterial peritonitis-related in-hospital mortality: applicable or not. J Gastroenterol Hepatol 2015; 30: 1205-10.

28. Na S, Yim S, Suh S, et al. ALBI versus Child-Pugh grading systems for liver function in patients with hepatocellular carcinoma. J Surg Oncol 2018; 117: 912-21.

29. Wang YY, Zhong JH, Su ZY, et al. Albumin-bilirubin versus ChildPugh score as a predictor of outcome after liver resection for hepatocellular carcinoma. Br J Surg 2016; 103: 725-34.

30. Ho SY, Liu PH, Hsu CY, et al. Comparison of twelve liver functional reserve models for outcome prediction in patients with hepatocellular carcinoma undergoing surgical resection. Sci Rep 2018; 8: 4773.

31. Shao L, Han B, An S, et al. Albumin-to-bilirubin score for assessing the in-hospital death in cirrhosis. Transl Gastroenterol Hepatol 2017; 2: 88.

32. Walayat $\mathrm{S}$, Martin D, Patel J, et al. Role of albumin in cirrhosis: from a hospitalist's perspective. J Community Hosp Intern Med Perspect 2017; 7: 8-14.

Received: 15.11 .2018

Accepted: 26.02.2019 